\title{
A experiência subjetiva do uso de psicotrópicos na perspectiva de pessoas com diagnóstico de esquizofrenia*1
}

\author{
lara Scaranelo Penteado Benini*2 \\ Erotildes Maria Leal*3
}

O presente artigo objetiva discutir a experiência subjetiva do uso de psicotrópicos na perspectiva de pessoas com o diagnóstico de esquizofrenia. Trata-se de um estudo qualitativo de base fenomenológica hermenêutica ou interpretativa que ocorreu a partir da análise de narrativas produzidas em grupos focais. Conclui ser a experiência do usuário de medicamentos diversa e importante, devendo ser incluida no processo de tomada de decisão quanto ao tratamento medicamentoso, onde o sujeito deve ser central.

Palavras-chave: Saúde Mental, psicotrópicos, experiência, esquizofrenia

*1 Artigo extraído, e reformulado, da dissertação de mestrado: A experiência subjetiva do uso de psicotrópicos na perspectiva de pessoas com o diagnóstico de esquizofrenia, Universidade Estadual de Campinas - Unicamp, 2015.

*2,3 Universidade Estadual de Campinas - Unicamp (Campinas, SP, Br). 


\section{ARTIGO}

\section{Introdução}

O presente artigo se propõe a discutir a experiência subjetiva do uso de psicotrópicos na perspectiva de pessoas com o diagnóstico de esquizofrenia. Trata-se de um estudo qualitativo de base fenomenológica hermenêutica ou interpretativa, que teve como matriz a pesquisa Experiência, narrativa e conhecimento: a perspectiva do psiquiatra $e$ a do usuário, que apresentava um conteúdo rico de narrativas acerca da experiência do uso de psicotrópicos, ainda não sistematicamente estudadas, e que ocorreu de forma multicêntrica nas cidades de Campinas, Salvador e Rio de Janeiro.

A pesquisa experiência foi desenvolvida no período de março de 2009 a fevereiro de 2011, a partir da parceria entre o Departamento de Saúde Coletiva da Faculdade de Ciências Médicas da Universidade Estadual de Campinas (DSC/FCM/UNICAMP), Instituto de Saúde Coletiva da Universidade Federal da Bahia (ISC/UFBA) e o Laboratório de Estudos e Pesquisas em Psicopatologia e Subjetividade do Instituto de Psiquiatria da Universidade Federal do Rio de Janeiro (IPUB/UFRJ). Seu financiamento ocorreu pelo CNPq e sua aprovação foi divulgada no edital MCT/CNPQ/CT-Saúde/MS/SCTIE/DECIT sob o n. 33/2008.

Apesar da pesquisa experiência não ter tido como objetivo principal conhecer a experiência do uso de psicotrópicos segundo a perspectiva do usuário, essa temática se destacou principalmente no momento em que se discutiu a experiência do tratamento como uma das dimensões da experiência de adoecimento. Sabemos que no contexto de tratamento, mesmo após as mudanças expressivas geradas pela Reforma Psiquiátrica Brasileira (RPB), a terapêutica medicamentosa manteve um lugar importante na sociedade e na cultura brasileira, sendo muitas vezes considerada a principal forma de cuidado quando não a única. Em um estudo sobre medicalização, em um serviço de saúde mental público, Ferrazza et al. (2013) constataram que $80 \%$ dos 430 sujeitos da amostra que deram entrada em um pronto atendimento 
foram encaminhados ao médico psiquiatra, sendo que mais de $99 \%$ dos que chegaram à consulta receberam prescrição de medicamentos psiquiátricos, em $78 \%$ dos prontuários não constava nenhum diagnóstico. Jenkins (2011), em seu trabalho sobre a existência de um eu farmacêutico (pharmaceutical self), cita uma pesquisa realizada no Brasil por João Biehl, onde aponta o contexto brasileiro como detentor de um sistema de saúde que se encontra em transformação pela economia neoliberal, em que a assistência psiquiátrica e a hospitalização têm sofrido uma expressiva redução em seu orçamento, enquanto há um aumento enfático na distribuição de psicotrópicos à população de nível econômico mais baixo. Segundo Jenkins (2011), para os psiquiatras, o grande problema da atualidade é a não adesão de alguns pacientes ao tratamento medicamentoso, mas para a antropologia, apesar da diminuição de hospitalização e sintomatologias, o problema está no fato de que as questões existenciais da vida subjetiva do indivíduo diagnosticado com transtorno mental permanecem sem melhoras com o uso de medicamentos psiquiátricos.

Neste artigo, buscamos compreender e enfatizar o papel do sujeito no uso de medicamentos, conferindo-lhe um lugar ativo, muitas vezes ignorado na prática clínica tão embebida de conhecimentos técnicos e científicos que delegam ao sujeito o lugar de passivo. Para tanto, discutimos o contexto brasileiro em que os medicamentos psiquiátricos se inserem e que interfere no cuidado prestado aos usuários de serviços de saúde mental.

\section{Medicamentos psiquiátricos e o contexto brasileiro: da verdade absoluta à contestação}

Com o advento da RPB, a internação foi um dispositivo de cuidado bastante contestado, o que notamos não ter acontecido com a prescrição de medicamentos psiquiátricos, uma terapêutica muito presente e pouco discutida no cuidado de pessoas em sofrimento mental. Em uma pesquisa realizada em trinta Centros de Atenção Psicossocial (CAPS) localizados na região Sul do Brasil, constatou-se que 91,8\% dos 1.067 usuários analisados faziam uso de algum psicofármaco (Kantorski et al., 2011). Em outra pesquisa realizada por Ferrazza et al. (2013, p. 263), eles observaram que o modelo psicossocial preconizado por nossas políticas públicas de saúde "ainda não conseguiu deslocar a hegemonia das práticas orientadas pelo modelo médico tradicional" e também manifestaram sua preocupação com a possibilidade de os medicamentos não serem mais apenas o principal ou muitas vezes o único recurso terapêutico do psiquiatra, mas que eles possam se tornar obrigatório mesmo que não necessário. 


\section{ARTIGO}

Sabemos que o problema de acesso ainda é presente em nosso país, apesar do "acesso universal" ser um dos princípios preconizados pelo Sistema Único de Saúde (Ministério da Saúde, 2004). Vieira (2010, p. 150) observa que no Brasil ainda não resolvemos o problema da indisponibilidade de medicamentos essenciais. Também é sabido haver problemas quanto ao acesso a serviços, se considerarmos os Centros de Atenção Psicossocial (CAPS) no censo Saúde Mental em Dados -8 que nos situa quanto à cobertura desses serviços no Brasil, sendo que em dezembro de 2010 ela era de $66 \%$, o que é considerado pelo Ministério da Saúde uma cobertura "boa", mas se olharmos para a cobertura dividida em regiões do Brasil, observa-se haver desigualdades entre as regiões; Centro-Oeste e o Norte, por exemplo, têm a pior cobertura considerada como "regular/ baixa" (Ministério da Saúde, 2011). Dessa forma, sem ignorar a importância que o acesso a serviços e medicamentos representa, fomos acometidos por algumas questões como: no campo da saúde mental, o uso de medicamentos psiquiátricos proporciona o melhor nível de saúde a todos os usuários? O prescritor de medicamentos, onde é possível acessar esses insumos, tem feito uma clínica centrada no sujeito e não na doença?

Nem todos os usuários de medicamentos os experienciam de forma positiva. Segundo os estudos de Mendes (2008), é "evidente um pensamento dualista, subjectivo e paradoxal em torno da medicação" (Mendes, 2008, p. 92). No entanto, apesar das evidências de que os usuários produzem diversas e distintas respostas quanto a sua experiência do uso de medicamentos (Read, 2009), encontramos que os usuários não se sentem em poder do conhecimento acerca dos psicotrópicos ou não são escutados quanto ao seu conhecimento/ experiência do uso, sendo a indagação de tomar ou não os medicamentos, ou quais medicamentos tomar, uma decisão unilateral (Read, 2009). Obviamente não se trata de abolir a medicação ou ignorar sua importância, comprovada nas pesquisas como benéfica para muitos usuários, mas de levantar a questão de que, quando a decisão de tomar esse ou outro medicamento psiquiátrico não passa pela experiência do sujeito que o toma, mesmo com toda boa intenção, podemos sem ao menos notar, colocá-lo no mesmo lugar de opressão e de descrédito que as antigas instituições psiquiátricas os colocaram, ou infelizmente ainda os colocam, podemos deixar de oferecer um tratamento que de fato seja sentido como positivo pelo usuário, podemos perder a oportunidade de desenvolver sujeitos autônomos e responsáveis quanto ao seu tratamento medicamentoso (Kantorski et. al., 2011), o que pode reverberar a outras instâncias da vida, entre tantas outras coisas tão importantes que podemos deixar de compreender e fazer com vistas ao melhor tratamento possível a essas pessoas. Se o sujeito em sofrimento mental não é desprovido de razão, ainda mais considerando que cada um tem a sua razão, seja ela qual for, por que não escutá-los também a respeito da sua experiência do 
uso de medicamentos? Se escutarmos, por que não considerar essa experiência com a mesma importância que consideramos o conhecimento científico acerca da psiquiatria e dos medicamentos psiquiátricos como dispositivo de tratamento?

Tenório (2002) aponta que uma das principais características do movimento da reforma psiquiátrica era a inclusão dos usuários e seus familiares nas discussões que cercavam esse processo de transformação dos serviços e do cuidado oferecido. Dessa forma, pensar em incluir o usuário nas decisões quanto a sua medicação é manter o espírito da reforma, mesmo porque o remédio parece ter mantido o seu lugar solitário de saber absoluto, destinado sempre e apenas aos sintomas. Nas palavras de Bissel, Ryan \& Morecroft (2006): “(...) estamos conscientes de que farmácia (...) opera a partir de um conjunto muito estreitamente definido de discursos, que são raramente ou quase nunca questionado ou desafiado, seja de dentro ou fora" (p. 57; tradução pessoal).

Considerando a RPB, o lugar de verdade incontestável que o medicamento ocupa e os usuários de serviços e de medicamentos psiquiátricos, buscamos compreender, no contexto da reformulação da assistência psiquiátrica, como os direitos e a proteção do sujeito em sofrimento mental se configuram a respeito do uso de medicamentos psiquiátricos no Brasil. Para tanto analisamos três documentos, o primeiro deles foi a Lei 10.216, que é tomado como um marco legislativo que institui a RPB. Essa lei versa sobre proteção e dos direitos das pessoas com problemas de saúde mental e reorganização do modelo de assistência. Em seu texto, no Art. $2^{\circ}$, é explanado o direito que o usuário, seu familiar ou responsável têm de receber todas as informações descritas no parágrafo único, em um atendimento de qualquer natureza, em saúde mental.

No número VIII do parágrafo único encontramos: "ser tratada em ambiente terapêutico pelos meios menos invasivos possíveis", ou no número I do mesmo parágrafo: "ter acesso ao melhor tratamento do sistema de saúde, consentâneo às suas necessidades". O que seria o tratamento menos invasivo para cada usuário do serviço de saúde mental? Ser medicado contra a sua vontade pode ser invasivo? Se o melhor tratamento é aquele que está em sintonia com as necessidades do sujeito, então pressupomos ser preciso escutá-lo, entendendo que sua necessidade talvez inclua não tomar medicamentos?

Embora essas questões possam emergir da leitura da Lei 10.216, ela não faz referência direta ao direito do usuário no que diz respeito aos medicamentos psiquiátricos. Sua formulação e aprovação se dão num contexto histórico particular. Era um momento em que as preocupações se dirigiam especialmente à exclusão, à cronificação, ao asilamento, que era o principal desafio a ser vencido àquela época. Os psicofármacos não foram citados, talvez por terem sido tomados como aliados do tratamento ambulatorial, mais humanizado e territorializado, o 


\section{ARTIGO}

que não deixa de ser em parte verdade. Segundo Read (2009) os medicamentos psiquiátricos são apenas uma das formas de ajudar as pessoas diagnosticadas com transtorno psiquiátrico, mas é sem dúvida, além do seu potencial lucrativo, a mais conveniente, visto que sua fabricação, assim como a sua distribuição são relativamente fáceis, podendo vislumbrar rapidamente os resultados. Nesse cenário, a desinstitucionalização era um assunto tão emergente que parece não ter havido espaço para pensar sobre a medicação, que é um agente poderoso que se coloca entre o médico e o usuário, outros profissionais de saúde mental e o usuário, a instituição de cuidado e o usuário, podendo estabelecer relações de poder tão verticalizadas quanto as das internações.

Passado mais de vinte anos de um processo de reforma que continua em aberto e em curso, talvez agora, porque já existe uma rede mínima de serviços comunitários, territoriais que lutam para instituir novas práticas de cuidado e que têm como propósito responder pela atenção também a pacientes graves, a desnaturalização do uso do medicamento psiquiátrico possa se dar.

O segundo documento considerado aqui é a Resolução CFM n. 1598/2000 publicada em 18 de agosto de 2000 e modificada pela Resolução CFM n. 1952/2010. Esse documento do Conselho Federal de Medicina, considerando os sujeitos diagnosticados com transtorno mental, normatiza seu atendimento médico. Encontramos em seu Art. $6^{\circ}$ a seguinte afirmação:

Nenhum tratamento deve ser administrado a paciente psiquiátrico sem o seu consentimento esclarecido, salvo quando as condições clínicas não permitirem a obtenção desse consentimento, e em situações de emergência, caracterizadas e justificadas em prontuário, para evitar danos imediatos ou iminentes ao paciente ou a outras pessoas. (Resolução CFM n. 1598/2000, 2000)

Segue em seu parágrafo único:

$\mathrm{Na}$ impossibilidade de obter-se o consentimento esclarecido do paciente, e ressalvadas as condições previstas no caput deste artigo, deve-se buscar o consentimento de um responsável legal. (Resolução CFM n. 1598/2000, 2000)

Dentre os documentos analisados, este é o único que refere o direito do sujeito diagnosticado com transtorno mental consentir ou não com um tratamento médico. Não especifica os medicamentos, mas indiretamente os inclui, já que a indicação desse tratamento é uma prerrogativa do médico. No entanto, além de ser uma resolução destinada aos médicos e não especificamente aos usuários, também se faz importante analisar que aqui se repete o mesmo impasse que se observa em relação à decisão dos usuários sobre a internação. A capacidade de os usuários de serviços de saúde mental tomarem decisões está, em tese, sob questão no caso da existência da doença mental. Isso não só entre profissionais 
como entre familiares. Isso para não citar a crença popular de que "lugar de louco é no hospício" (Tenorio, 2002, p. 34), que não raro se associa a uma outra, que pode ser tão prejudicial quanto a primeira, de que todo "louco" sem medicação representa perigo à sociedade. Será que podemos pensar que a mesma redução observada no passado, que igualou tratamento à internação, hoje se dá com a medicação através da substituição da internação pela medicação? A internação foi posta sob questão e a naturalização da sua indicação como recurso único ou preferencial para o tratamento das pessoas diagnosticadas com transtorno mental foi interrogada. Considerando as pesquisas citadas acima, caberia perguntar se a medicação a substituiu neste lugar? Parece que sim.

O terceiro e último documento analisado foi a cartilha Direito à Saúde Mental do Ministério Público Federal/Procuradoria Federal dos Direitos do Cidadão. Esta cartilha teve a colaboração de diversos conselhos federais que regulamentam a profissão da maioria dos profissionais que compõe a equipe multidisciplinar dos Centros de Atenção Psicossocial (CAPS), e dos demais dispositivos que compõem a rede de atenção à saúde mental, a saber: psicologia, medicina, serviço social e enfermagem.

A cartilha é dividida em temas que abarcam perguntas organizadas em itens. No tema Saúde Integral encontramos a seguinte pergunta no item 7: "Os remédios causam dependência?'. Segue sua resposta:

Não, apenas um grupo específico de medicamentos é capaz de provocar alguma dependência, e o médico está sempre alerta para essa possibilidade. Os remédios, quando bem utilizados, podem ajudar não apenas no controle de crises agudas, como são ainda fundamentais na efetividade do tratamento de longo prazo de casos mais severos. (Cartilha: Direito à Saúde Mental, p. 15)

Ao afirmar a existência de um grupo de medicamentos com potencial em gerar dependência, a cartilha não se preocupa em especificá-los. Não sabemos a que grupo se refere, mas é possível supor que sejam aos tranquilizantes, ou ansiolíticos. Sabe-se, entretanto, que os problemas que podem decorrer do uso regular dos medicamentos psiquiátricos não são apenas a síndrome de dependência, em seu sentido médico. O estudo All you need to know? (SAMH, 2004), citado por Read (2009), refere os antidepressivos do grupo dos SSRIs (inibidores seletivos da receptação de serotonina) como medicamentos com alta probabilidade de provocar efeitos adversos quando se tenta parar de tomá-los. Alguns deles têm risco significativo de provocar síndrome de abstinência. Além disso, ele afirma serem muitos os efeitos adversos dos medicamentos psiquiátricos em geral, e que pode haver dificuldade entre os usuários de medicamentos em distinguir o que são sentimentos ligados a sua própria condição e o que são sentimentos ligados a esses efeitos. 


\section{ARTIGO}

$\mathrm{Na}$ resposta dada pela cartilha, também não são citados os efeitos colaterais já conhecidos, tampouco se fala sobre a possibilidade de cada sujeito experienciar os medicamentos de maneiras distintas e que essas experiências são importantes para negociar com seu médico os medicamentos ou o tratamento que lhe faça se sentir melhor. Além disso, a cartilha afirma estar o médico sempre atento ao risco da produção de dependência. Toda a responsabilidade e todo o conhecimento sobre o tratamento medicamentoso ficam assim concentrados na pessoa do médico. Aqui se vê, entre médico e usuário, uma relação verticalizada, em que cabe pouco a negociação, já que para isso é preciso que haja, no mínimo, a suposição de que o usuário também possui algum conhecimento sobre o uso dos seus medicamentos ou a eles relacionado, que é tão importante quanto o conhecimento técnico/científico do médico, e que deve ser considerado.

No tema Exames e Prontuários/Atendimento encontramos a seguinte pergunta no item 52: "A pessoa com transtorno mental tem direito a recusar o tratamento?". Ela é seguida da resposta:

Poderá consentir ou recusar, de forma livre, voluntária ou esclarecida, com adequada informação, sobre procedimentos diagnósticos e/ou terapêuticos e cirúrgicos a que será submetido, para os quais deverá conceder autorização por escrito, por meio do termo de consentimento esclarecido. A única exceção é se estiver em surto agudo e, nestes episódios, algum familiar deverá assumir sua autonomia. (Cartilha: Direito à Saúde Mental, p. 31)

A aceitação ou a recusa por meio de um consentimento esclarecido quando se trata de terapêuticas medicamentosas ainda é, a meu ver, um ponto intocado. Aqui observamos uma questão importante que é a afirmação do direito de ser informado adequadamente quanto à terapêutica que lhe será aplicada. Para tanto, as relações horizontalizadas, que supõem que estão em questão dois sujeitos que portam saberes, embora diferenciados, são de extrema importância.

A partir dessa simples análise, podemos afirmar o quanto o uso e a eficácia dos medicamentos permanecem naturalizados como verdades absolutas e incontestáveis. Nenhum dos três documentos analisados se abriu à experiência dos usuários, tampouco os inclui no processo de tomada de decisão quanto ao tratamento medicamentoso, e quando os incluem sempre mantêm a ressalva do quanto pode ser necessária a tomada de decisão por outros. Não há uma preocupação em orientá-los quanto aos seus direitos em relação ao uso de psicotrópicos ou o quão importante pode ser aquilo que sentem e constroem a partir do uso de medicamentos. O conhecimento acerca dos medicamentos, produzido na e pela indústria farmacêutica, e supostamente com base na ciência e na tecnologia, parece ainda dominar todo o processo da terapêutica medicamentosa. 


\section{Resultados e Conclusão}

A partir da análise das narrativas nos três campos de pesquisa (Rio de Janeiro, Campinas e Salvador), podemos afirmar que os usuários de psicotrópicos muito têm a dizer sobre suas experiências acerca do tratamento medicamentoso, e essa experiência se apresenta diversa e não descolada do contexto em que ela se insere. A forma como cada sujeito experiencia a medicação está vinculada à maneira como cada sujeito compreende aquilo que está associado ao uso de psicotrópicos, por exemplo, o diagnóstico e a medicação, que apareceram nas narrativas entendidos como interdependentes e, por isso, a forma que o sujeito experiência o diagnóstico interferindo na experiência do uso de medicação. Também observamos ser a minoria que vivencia o uso de medicamentos como uma escolha feita diante de seu diagnóstico; a maioria vivencia esse uso como uma imposição familiar, social e da própria equipe de cuidado, tendo aqueles que acatam mesmo se sentindo "cobaia" de uma ciência que ainda não descobriu o remédio correto e outros que passam a administrar os medicamentos a partir de uma lógica própria. O contexto do hospital psiquiátrico parece ser o que mais abarca experiências negativas acerca da medicação, principalmente quando se trata da medicação injetável, mas a relação entre médico e usuário também exerce fortes influências na maneira pela qual o sujeito passa a vivenciar o uso de seus medicamentos.

Há narrativas que evidenciam o medicamento como capaz de remitir os sintomas, diminuir sua intensidade ou até mesmo mantê-los sem nenhuma alteração, e podemos observar o mesmo quanto à crise. A experiência dos efeitos indesejáveis também são diversas, e essa vivência é um dos motivos que levam os sujeitos a parar com o uso de medicação, mas há narrativas que evidenciam a experiência do remédio como quem assume o controle da sua vida se colocando como coadjuvante nesse processo, sendo o contrário também verdadeiro, e outras que apontam o remédio como impeditivo de algumas atividades cotidianas, do trabalho, e de relação sexual. Também observamos narrativas que vivenciam os medicamentos como produtores de preconceito; mesmo quando geram bons resultados quanto aos sintomas, não resolvem problemas sociais gerados pelo simples fato de se estar tomando remédios psiquiátricos.

Como podemos observar, o uso de medicamentos abarca experiências diversas e de forte influência na maneira pela qual o sujeito compreende e executa seu tratamento. Os documentos destinados à RPB, aos médicos e aos usuários não se ocuparam dessas questões, e o que vemos na prática ainda é um impasse pouco discutido quanto ao tratamento medicamentoso. Essa discussão se faz necessária e importante para que possamos oferecer tratamentos mais adequados e eficazes em saúde mental, preocupados em inserir os sujeitos que deles usufruem nos debates quanto ao processo de tomada de decisão, ocupando-se de uma clínica centrada 


\section{ARTIGO}

no sujeito e não na doença, já que fica evidente o quanto o acesso a serviços e medicamentos em saúde mental não garante uma experiência positiva de seus usuários com o uso desses agentes, nem tampouco garante uma continuidade desse tratamento conforme prescrito pelos médicos.

\section{Referências}

Bissel, P.; Ryan, K. \& Morecroft C. (2006). Narratives about illness and medication: a neglected theme/new methodology within pharmacy practice research. Part I: Conceptual Framework. Pharm World SCI. 28, 54-60.

Brasil. Ministério da Saúde. Saúde Mental em Dados - 8, ano VI, n. 8. Informativo eletrônico. Brasília: janeiro de 2011. Recuperado em $1^{\circ}$ ago 2014, de: <http://www.bvsms. saude.gov.br/bvs/periodicos/saude_mental_dados_v8.pdf $>$.

Congresso Nacional. Lei 10.216, de 6 de abril de 2001. Recuperado em 9 maio 2014, de: $<$ http// www.planalto.gov.br/ccivil-03/leis/leis-2001/102016.htm>.

Conselho Federal de Medicina. Resolução CFM no 1598/200. Recuperado em 9 maio 2014, de: <http//www. portalmedico.org.br/resoluções/cfm/2000/1598-2000.htm>.

Ferrazza, D.A.; Rocha, L.C. \& Luzio, C.A. (2013, jul-dez). Medicalização em um serviço público de saúde mental: um estudo sobre a prescrição de psicofármacos. Revista Internacional de Psicologia, 6(2), 255-265.

Jenkins, J.H. (2011). Psychopharmaceutical self and imaginary in the social field of psychiatric treatment. In Jenkins, J.H. (Org.). Pharmaceutical Self: The Global Shaping of Experience in an age of Psychopharmacology (pp. 17-40). Santa Fe, New Mexico: Sarpress.

Kantorski, L.P.; Jardim, V.M.R.; Porto, A.R.; Schek, G.; Cortes, J.M. \& Oliveira, M.M. (2011). Descrição de oferta e consumo dos psicofármacos em Centros de Atenção Psicossocial na região Sul brasileira. Revista Escola de Enfermagem da USP, 45(6), 1481-1487.

Mendes, C.S.A. (2008). Recovery e medicação: perspectivas e percepções de indivíduos com experiência de doença mental. Dissertação. Lisboa: Instituto Superior de Psicologia Aplicada - ISPA.

Ministério Público Federal/Procuradoria Federal dos Direitos do Cidadão. Cartilha: Direito à Saúde Mental. Recuperado em 9 maio 2014, de: <http://www.pfdc.pgr.mpf.br/ atuacao-e-conteudos-de-apoio/publicacoes/saude-mental/direito-saude-mental-2012/>.

Read, J. (2009). Psychiatric Drugs: Key Issues and Service User Perspectives. Mind National Association for Mental Health. Londres: Palgrave Macmillan.

Serpa Junior, O.D. (2011, fev.). Experiência, narrativa e conhecimento: a perspectiva do psiquiatra e a do usuário. Relatório Técnico Final. Número do processo: 575257/20083. Edital MCT/CNPq/CT-Saúde/MS/SCTIE/DECIT n. 33/2008. Rio de Janeiro. 
Tenório, F.A. (2002.jan./abr.). Reforma Psiquiátrica Brasileira, da década de 1980 aos dias atuais: história e conceitos. História, Ciência, Saúde-Manguinhos, RJ, 9(1), 25-59.

Vieira, F.S. (2010). Assistência farmacêutica no sistema público de saúde no Brasil. Revista Panam Salud Publica, 27(2), 149-56.

\section{Resumos}

(The subjective experience of using psychotropic drugs from the perspective of people diagnosed with schizophrenia)

This paper aims to discuss the subjective experience of using psychotropic drugs from the perspective of people diagnosed with schizophrenia. This is a qualitative study, with a hermeneutic or interpretative phenomenological base, that resulted from analyzing narratives produced in focus groups. It concludes that the experience of drug users is diverse and important, and should be included in the decision-making process as to their drug treatment, in which the subject must be central.

Key words: Mental Health, psychotropic drugs, experimence, schizophrenia

(L'expérience subjective de l'usage de substances psychotropes du point de vue de personnes diagnostiquées schizophrènes)

Cet article vise à discuter de l'expérience subjective de l'usage de substances psychotropes du point de vue de personnes diagnostiquées schizophrènes. Il s'agit d'une étude qualitative fondée sur une base phénoménologique herméneutique ou interprétative qui a été réalisée à partir de l'analyse de récits produits dans des groupes de discussion. J'en ai conclu que l'expérience de l'utilisateur de médicaments est variée et importante, et devrait être incluse dans le processus décisionnel concernant le traitement médicamenteux, où le sujet doit être central.

Mots-clés: Santé mentale, psychotropes, expérience, schizophrénie

(La experiencia subjetiva del uso de drogas psicotrópicas en la perspectiva de personas con el diagnóstico de esquizofrenia)

Este artículo tiene como objetivo discutir la experiencia subjetiva del uso de psicotrópicos en la perspectiva de las personas con un diagnóstico de esquizofrenia. Se trata de un estudio cualitativo con base fenomenológica hermenéutica o interpretativa que se dio desde el análisis de narrativas producidas en grupos focales. Concluye que la experiencia del usuario de drogas es diversa e importante, y debe ser incluida en el proceso de toma de decisiones en cuanto al tratamiento medicamentoso, en el que el sujeto debe ser central.

Palabras clave: Salud mental, drogas psicotrópicas, experimento, esquizofrenia 


\section{ARTIGO}

(Die subjektive Erfahrung der Benutzung von Psychopharmaka von mit Schizophrenie diagnostizierten Personen)

Dieser Artikel untersucht die subjektiven Erfahrungen, die bei der Benutzung von Psychopharmaka entstehen, von Personen, die mit Schizophrenie diagnostiziert wurden. Diese qualitative Studie basiert auf der phänomenologischen oder interpretativen Hermeneutik und wurde mittels einer Analyse von Erzählungen aus Fokusgruppen durchgeführt. Die Resultate zeigen, dass die Erfahrungen der Medikamentenbenutzer vielfältig und relevant sind, weshalb sie im Entscheidungsprozess der medikamentösen Therapie einbezogen werden sollten, um somit dem Patienten eine zentrale Rolle zu erteilen.

Stichwörter: Geistige Gesundheit, Psychopharmaka, Erfahrungen, Schizophrenie

(精神分裂症病人服用神经控制药物的主观感受)

本论文讨论了精神分裂症病人在使用脑神经控制药物时的主观感受，分析 了病人单独或者在小组里谈论并且经由研究人员记录下来的有关他们服用脑神 经控制药品之后的经历。本论文目的是分析病人服药之后的感受。作者的结论 是, 每个病人服用之后的感受都不同, 医生在给病人配药时应该考虑到病人的 感受，因为病人是主体。

关键词: 精神健康, 脑控制药物, 感受, 精神分裂症。

Citação/Citation: Benini, I.S.P., Leal, E.M. (2016, março). A experiência subjetiva do uso de psicotrópicos na perspectiva de pessoas com o diagnóstico de esquizofrenia. Revista Latinoamericana de Psicopatologia Fundamental, 19(1), 30-42.

Editores do artigo/Editors: Prof. Dr. Manoel Tosta Berlinck e Profa. Dra. Sonia Leite

Recebido/Received: 25.11.2015/ 11.25.2015 Aceito/Accepted: 14.12.2015 / 12.14.2015

Copyright: (C) 2009 Associação Universitária de Pesquisa em Psicopatologia Fundamental/ University Association for Research in Fundamental Psychopathology. Este é um artigo de livre acesso, que permite uso irrestrito, distribuição e reprodução em qualquer meio, desde que o autor e a fonte sejam citados / This is an open-access article, which permits unrestricted use, distribution, and reproduction in any medium, provided the original authors and sources are credited. 
Financiamento/Funding: As autoras declaram não ter sido financiadas ou apoiadas / The authors have no support or funding to report.

Conflito de interesses/Conflict of interest: As autoras declaram que não há conflito de interesses / The authors have no conflict of interest to declare.

\section{Iara Scaranelo Penteado Benini}

Psicóloga; Especialista em Saúde Mental em Saúde Coletiva. Mestre em Saúde Coletiva, na área de concentração Política, Planejamento e Gestão, pelo Departamento de Medicina Preventiva e Social da Faculdade de Ciências Médicas da Universidade Estadual de Campinas - Unicamp (Campinas, SP, Br).

\section{Erotildes Maria Leal}

Médica; Mestre em Saúde Coletiva pela Universidade do Estado do Rio de Janeiro UERJ (Rio de Janeiro, RJ, Br) e Doutora em Psiquiatria e Saúde Mental pela Universidade Federal do Rio de Janeiro - UFRJ (Rio de Janeiro, RJ, Br); pós-doutorado "International Research Capacity-Building Program for Health Related Professional to Study the Drug Phenomenon in Latin America" - Universidade de Toronto/CICAD; Professora adjunta do curso de Medicina da UFRJ, campus de Macaé (RJ) e professora associada da pós-graduação em Saúde Coletiva da Faculdade de Ciências Médicas Universidade Estadual de Campinas - Unicamp (Campinas, SP, Br), linha de pesquisa "Gestão e subjetividade: estudos das práticas e políticas de saúde".

Rua Tessália Vieira de Camargo, 126 - Cidade Universitária “Zeferino Vaz"

Grupo de pesquisa Saúde Coletiva e Saúde Mental: Interfaces

13083-887 Campinas, SP, Br.

e-mail: eroleal@gmail.com

This is an open-access article, which permits unrestricted use, distribution, and reproduction in any medium for non-commercial purposes provided the original authors and sources are credited. 